\title{
Study for Correlation between Objective and Subjective Voice Parameters in Patients with Dysphonia
}

\author{
Jung Woo Park (D), Boram Kim (iD), Jae Hwan Oh (D), \\ Tae Kyu Kang (D), Dong Young Kim (D), and Joo Hyun Woo (D)
}

Department of Otorhinolaryngology-Head and Neck Surgery, Gachon University College of Medicine, Gil Medical Center, Incheon, Korea

발성장애 환자에서 주관적 음성검사와 객관적 음성검사의 연관성 연구

박정우, 김보람, 오재환, 강태규, 김동영, 우주현

가천대학교 의과대학 길병원 이비인후과학교실

Background and Objectives Voice evaluation is classified into subjective tests such as auditory perception and self-measurement, and objective tests such as acoustic and aerodynamic analysis. When evaluating dysphonia, subjective and objective test results do not always match. The purpose of this study was to analyze the relationship between subjective and objective evaluation in patients with dysphonia and to identify meaningful parameters by disease.

Materials and Method The total of 322 patients who visited voice clinic from May 2017 to May 2018 were included in this study. Laryngeal lesions were identified using stroboscopy. Pearson correlation test was performed to analyse correlation between subjective tests including GRBAS scale and voice handicap index, and objective tests including jitter, shimmer, noise to harmonic ratio (NHR), cepstral peak prominence (CPP), maximal phonation time (MPT), mean flow rate, and subglottic pressure.

Results In vocal nodule and sulcus vocalis, among GRBAS system, grade and breathiness showed good correlation with CPP, and roughness showed good correlation with jitter or shimmer. In unilateral vocal cord paralysis (UVCP), grade and breathiness showed a very good correlation with CPP, and also good correlation with jitter, shimmer, NHR, and MPT. Also asthenia showed good correlation with CPP and MPT. Vocal polyp has a limited association with other diseases. Conclusion In patients with dysphonia, grade and breathiness showed good correlation with CPP, jitter, and shimmer, and reflect the state of voice change well especially in UVCP, CPP, and MPT.

Keywords Voice; Evaluation; Acoustics; Auditory-perception; Correlation.

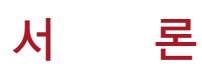

음성검사는 발성장애 환자에서 목소리의 질을 평가하고 이를 바탕으로 치료 계획을 수 립하고 치료 결과에 대한 피드백을 제공하는 데 사용된다. 주관적 검사로는 청지각적검 사와 자가측정검사가 있으며 객관적 검사로는 음향검사, 공기역학적검사 등이 있다. ${ }^{1)}$ 각 각의 검사가 독립적으로 의미를 가지지만 주관적 검사와 객관적 검사의 결과가 항상 일 관성 있게 나타나지는 않으며 이런 경우 평가 분석에 혼선이 빚어지기도 한다. 예전부터
Received November 25, 2019

Revised December 3, 2019

Accepted December 6, 2019

Corresponding Author

Joo Hyun Woo, MD, PhD

Department of OtorhinolaryngologyHead and Neck Surgery,

Gachon University

College of Medicine,

Gil Medical Center,

21 Namdong-daero 774beon-gil, Namdong-gu, Incheon 21565, Korea

Tel $+82-32-460-3765$

Fax +82-32-467-9044

E-mail woojh@gilhospital.com

ORCID iDs

Jung Woo Park (D)

https://orcid.org/0000-0001-5082-7530

Boram Kim (D)

https://orcid.org/0000-0003-4035-0040 Jae Hwan Oh (D)

https://orcid.org/0000-0002-1180-2581

Tae Kyu Kang (iD

https://orcid.org/0000-0002-7274-0476

Dong Young Kim (D)

https://orcid.org/0000-0001-5485-7198

Joo Hyun Woo (D)

https://orcid.org/0000-0002-8584-563X

This is an Open Access article distributed under the terms of the Creative Commons Attribution Non-Commercial License (https://creativecommons.org/ licenses/by-nc/4.0) which permits unrestricted non-commercial use, distribution, and reproduction in any medium, provided the original work is properly cited. 
많은 연구에서 객관적 검사들과 주관적 검사들 간의 연관성을 찾고자 하는 노력이 있었다. ${ }^{2-4)}$ 하지만 아직 객관적인 음성평 가 항목들과 주관적임 음성평가 항목들 간의 전체적인 연관성 을 분석한 보고는 없으며 동일환 환자군에서 이러한 분석을 통해 검사들 사이의 연관성을 밝혀낼 수 있다면 좀 더 임상적 인 의미를 둘 수 있을 것으로 판단된다. 이에 저자들은 발성 장애 환자들에서 주관적 평가와 객관적 평가 사이의 전체적 인 연관성을 분석하여 질환별로 의미 있는 평가 요소들을 알 아보고자 하였다.

\section{대상 및 방법}

대 상

2017년 5월 2018년 5월까지 발성장애로 가천대 길병원에 내 원하여 음성검사를 시행한 환자들을 대상으로 하였고, 연구 는 가천대 길병원 임상연구윤리센터의 허가를 받아 진행되었 다(GFIRB2019-424). 후두 스트로보스코피를 이용하여 성대 병변을 확인하였다. 모든 환자들에 대하여 GRBAS 척도를 이 용한 청지각적검사, 음성장애지수검사, 음향학적검사, 공기역 학적 검사를 시행하였다. 음향검사로는 기본주파수(fundamental frequency), 주파수변동율(jitter), 진폭변동율(shimmer), 잡음 대 배음비(noise to harmonic ratio, NHR), 켑스트 럴 최대융기(cepstral peak prominence, $\mathrm{CPP}$ )가 시행되었다. 공기역학적 검사로는 최대발성시간(maximal phonation time), 평균호기류율(mean flow rate), 성문하압(subglottic pressure) 이 사용되었다.

\section{음성검사}

음향학적 검사는 Computerized Speech Lab(CSL, Kay Elemetrics, Montvale, NJ, USA)의 프로그램인 다면음성프로 그램(Multi-Dimensional Voice Program, MDVP)을 사용하 여 기본주파수, 주파수변동율, 진폭변동율, NHR을 측정하였 다. 마이크를 입에서 $3 \sim 5 \mathrm{~cm}$ 간격을 두고 편안한 자세에서 환 자가 평소 사용하는 편안한 목소리로 /아/ 발성을 5 초 이상, 평 균 3회 정도 반복하여 측정하였다. 또한 음성분석 프로그램에 저장된 환자의 음성파일을 이용하여 Hillenbrand 등희의 방법 에 따라 켑스트럴최대융기-연장모음발성 $(\mathrm{CPP}-\mathrm{a} /, \mathrm{CPP}-\mathrm{a})$ 과 켑스트럴최대융기-연속발화(CPP-speech, $\mathrm{CPP}-\mathrm{S})$ 분석을 시 행하였다.

공기역학적 검사는 Phonatory Aerodynamic System(Model 6600 , Kay Elemetrics)을 사용하여, 최대발성시간, 평균호기 류율, 성문하압을 측정하였다. 편안한 자세에서 편안한 목소 리로 마스크를 착용하여 평균 3회 정도 실시하였다.
청지각적검사는 GRBAS 척도를 사용하였으며 숙련된 음 성치료사 1 인이 환자의 음성을 청취한 후 판단하였다. 각각의 음성에 대해서 전체음질(grade, $\mathrm{G}$ ), 거친음(roughness, R), 기 식음(breathiness, B), 무력음(asthenia, A), 긴장음(strain, S) 등에 대해서 정상인 0 점에서 가장 나쁜 3점까지 측정하였다.

음성장애지수(Voice Handicap Index)는 한국어판 음성장 애지수를 사용하였다. ${ }^{6}$ 주관적 검사인 GRBAS 척도 그리고 음성장애지수와 객관적 검사 항목인 켑스트럴최대융기-연장 모음발성 $(\mathrm{CPP}-\mathrm{a})$, 켑스트럴최대융기-연속발화 $(\mathrm{CPP}-\mathrm{s})$, 기본 주파수, 주파수변동율, 진폭변동율, $\mathrm{NHR}$, 최대발성시간, 평균 호기류율, 성문하압 사이의 연관성을 측정하였다.

\section{통계 분석}

GraphPad Prism 5(GarphPad Software, Inc., La Jolla, CA, USA) 프로그램의 Pearson correlation test를 이용하여 각 항목들간의 연관성을 측정하였다. 양방향 상관관계 분석에 서 $\mathrm{p}<0.05$ 를 유의미한 것으로 하였으며, 상관계수가 0.3 미 만은 상관관계 없음, 0.3 0.5는 낮은 연관성(fair), 0.5 0.7은 중등도의 연관성(good), 0.7 0.9는 높은 연관성(very good) 이 있는 것으로 정의하였다. ${ }^{7}$

\section{결 과}

344 명의 환자가 평가 기간 동안 음성검사를 시행받았으며 그중 이전에 음성질환으로 치료를 받은 병력이 있는 22명을 제외한 322 명의 환자가 평가에 포함되었다. 남자는 149 명이었 으며 여자는 173명이었다. 질환별로 구분하였을 때 성대용종 과 성대구증 환자들이 각각 69명으로 가장 많았으며 그 뒤를

Table 1. Demographic data of patients and disease distribuation

\begin{tabular}{lc}
\hline Gender (M/F) & $149 / 173$ \\
Age (mean \pm standard deviation) & $52.08 \pm 13.39$ \\
Vocal nodule (n) & 58 \\
Vocal polyp (n) & 69 \\
Unilateral vocal cord paralysis (n) & 30 \\
Vocal paresis (n) & 7 \\
Sulcus vocalis (n) & 69 \\
Intracordal cyst (n) & 13 \\
Functional dysphonia (n) & 16 \\
Glottic cancer (n) & 11 \\
Leukoplakia (n) & 11 \\
Granuloma (n) & 7 \\
Reinke's edema (n) & 10 \\
Laryngeal papilloma (n) & 8 \\
Others (n) & 13 \\
Total (n) & 322 \\
\hline
\end{tabular}


이어서 성대결절 환자가 58 명, 일측성 성대마비 환자가 30 명 이 었다(Table 1).

전체 환자를 대상으로 한 분석에서 음성장애지수는 객관 적인 평가 항목들과 연관성이 없었으나 전체음질과 기식음은 $\mathrm{CPP}-\mathrm{a}, \mathrm{CPP}-\mathrm{s}$, 주파수변동율, 진폭변동율, $\mathrm{NHR}$, 최대발성 시간 등과 중등도의 연관성을 보였다(Table 2).

성대점막 질환별로 구분해서 살펴보면 69명의 성대용종
환자들을 분석하였을 때 전체음질은 주파수변동율과 중등도 의 연관성이 있었고 긴장음은 진폭변동율과 중등도의 연관 성이 있었다(Table 3). 69명의 성대구증 환자들에서는 전체음 질과 기식음에서 동일하게 $\mathrm{CPP}-\mathrm{a}, \mathrm{CPP}-\mathrm{s}$, 그리고 주파수변 동율에서 중등도의 연관성을 보였으며, 전체음질과 거친음에 서 진폭변동율와 중등도의 연관성을 보였다(Table 4). 58명 의 성대결절 환자들을 분석하였을 때 전체음질과 기식음은

Table 2. Correlation between subjective and objective parameters in total patients $(n=322)$

\begin{tabular}{lllllll} 
& VHI & G & R & B & A & S \\
CPP-a & -0.276 & $-0.634^{*}$ & -0.396 & $-0.588^{*}$ & -0.472 & $-0.148^{*}$ \\
CPP-s & $-0.137^{*}$ & $-0.158^{*}$ & -0.054 & $-0.126^{*}$ & $-0.157^{*}$ & $-4.343^{*}$ \\
\hline F0 & $0.127^{*}$ & $0.163^{*}$ & -0.0003 & $0.159^{*}$ & $0.204^{*}$ & $0.059^{*}$ \\
Jitter & $0.2537^{*}$ & $0.675^{*}$ & 0.408 & $0.639^{*}$ & 0.442 & 0.237 \\
Shimmer & $0.262^{*}$ & $0.621^{*}$ & 0.504 & $0.536^{*}$ & 0.300 & 0.376 \\
NHR & $0.212^{*}$ & $0.563^{*}$ & 0.301 & $0.529^{*}$ & 0.315 & 0.375 \\
\hline MPT & $-0.193^{*}$ & $-0.536^{*}$ & -0.306 & $-0.513^{*}$ & -0.423 & $-0.124^{*}$ \\
MFR & 0.053 & 0.401 & $0.160^{*}$ & 0.436 & 0.317 & $0.087^{*}$ \\
SP & -0.025 & 0.347 & 0.293 & 0.336 & -0.081 & 0.434 \\
\hline
\end{tabular}

${ }^{*} \mathrm{p}<0.05$. VHI: Voice Handicap Index, G: grade, R: rounghness, B: breathiness, A: asthenic, S: strained, CPP-a: cepstral peak prominence in /a/, CPP-s: cepstral peak prominence in sentence reading, F0: fundamental frequency, NHR: noise to harmonic ratio, MPT: maximal phonation time, MFR: mean flow rate, SP: subglottic pressure

Table 3. Correlation between subjective and objective parameters in vocal polyp patients $(n=69)$

\begin{tabular}{lcccccc} 
& VHI & G & R & B & A & S \\
CPP-a & -0.213 & $-0.320^{*}$ & $-0.250^{*}$ & -0.200 & -0.133 & -0.115 \\
CPP-s & -0.143 & 0.095 & 0.109 & 0.157 & -0.059 & 0.126 \\
F0 & -0.019 & 0.114 & -0.051 & 0.043 & 0.190 & -0.165 \\
Jitter & $0.268^{*}$ & $0.511^{*}$ & 0.489 & $0.295^{*}$ & 0.102 & 0.428 \\
Shimmer & 0.198 & 0.412 & 0.444 & 0.162 & -0.066 & $0.533^{*}$ \\
NHR & 0.209 & $0.341^{*}$ & $0.352^{*}$ & 0.079 & -0.113 & 0.467 \\
MPT & 0.034 & -0.356 & -0.180 & -0.451 & -0.079 & -0.230 \\
MFR & -0.191 & 0.158 & -0.005 & $0.279^{*}$ & -0.112 & $0.274^{*}$ \\
SP & 0.018 & 0.321 & $0.262^{*}$ & $0.373^{*}$ & -0.003 & 0.412 \\
\hline
\end{tabular}

*p<0.05. VHI: Voice Handicap Index, G: grade, R: rounghness, B: breathiness, A: asthenic, S: strained, CPP-a: cepstral peak prominence in /a/, CPP-s: cepstral peak prominence in sentence reading, F0: fundamental frequency, NHR: noise to harmonic ratio, MPT: maximal phonation time, MFR: mean flow rate, SP: subglottic pressure

Table 4. Correlation between subjective and objective parameters in sulcus vocalis patients $(n=69)$

\begin{tabular}{lccccccc} 
& VHI & G & R & B & A & S \\
\hline CPP-a & 0.014 & $-0.638^{*}$ & $-0.452^{*}$ & $-0.559^{*}$ & $-0.387^{*}$ & -0.022 \\
CPP-s & -0.098 & $-0.640^{*}$ & $-0.473^{*}$ & $-0.564^{*}$ & $-0.325^{*}$ & -0.001 \\
F0 & 0.093 & 0.212 & 0.171 & $0.264^{*}$ & $0.302^{*}$ & -0.036 \\
Jitter & 0.041 & $0.621^{*}$ & $0.472^{*}$ & $0.522^{*}$ & $0.309^{*}$ & 0.115 \\
Shimmer & 0.047 & $0.536^{*}$ & $0.543^{*}$ & $0.455^{*}$ & 0.209 & 0.228 \\
NHR & 0.087 & 0.111 & 0.016 & 0.116 & 0.077 & $0.279^{*}$ \\
MPT & 0.010 & $-0.452^{*}$ & $-0.328^{*}$ & $-0.499^{*}$ & $-0.294^{*}$ & -0.120 \\
MFR & -0.033 & $0.423^{*}$ & $0.353^{*}$ & $0.424^{*}$ & -0.004 & $0.278^{*}$ \\
\hline SP & 0.104 & $0.383^{*}$ & $0.338^{*}$ & $0.442^{*}$ & -0.110 & $0.361^{*}$
\end{tabular}

${ }^{*} \mathrm{p}<0.05$. VHI: Voice Handicap Index, G: grade, R: rounghness, B: breathiness, A: asthenic, S: strained, CPP-a: cepstral peak prominence in /a/, CPP-s: cepstral peak prominence in sentence reading, F0: fundamental frequency, NHR: noise to harmonic ratio, MPT: maximal phonation time, MFR: mean flow rate, SP: subglottic pressure 
$\mathrm{CPP}-\mathrm{a}, \mathrm{CPP}-\mathrm{s}$ 와 중등도의 연관성을 보였으며 전체음질과 거 친음은 주파수변동율, 진폭변동율과 중등도의 연관성을 보 였다(Table 5).

30 명의 일측성 성대마비 환자를 분석하였을 때 전체음질은 $\mathrm{CPP}-\mathrm{a}, \mathrm{CPP}-\mathrm{s}$, 최대발성시간과 높은 연관성을 보였으며 주 파수변동율, 진폭변동율, NHR과 중등도의 연관성을 보였다. 기식음은 $\mathrm{CPP}-\mathrm{s}$ 와 높은 연관성을 보였으며 $\mathrm{CPP}-\mathrm{a}$, 주파수 변동율, 진폭변동율, $\mathrm{NHR}$, 최대발성시간, 평균호기류율 등과 중등도의 연관성을 보였다. 무력음도 $\mathrm{CPP}-\mathrm{a}, \mathrm{CPP}-\mathrm{s}$, 최대발 성시간 등과 중등도의 연관성을 보였으며 긴장음은 $\mathrm{NHR}$ 과 중등도의 연관성을 보였다(Table 6).

음성장애지수는 대부분의 객관적인 평가 항목들과 연관성 을 보이지 못했다.

\section{고 찰}

이번 연구는 음성장애 환자에서 시행되는 객관적인 평가 항 목들과 주관적인 평가 항목들 사이의 연관성을 알아보고자 시행되었다.
이전의 음향학적 평가와 청지각적 평가의 연관성을 분석한 연구에 따르면 거친음(R)은 주파수변동율이 높고, 기본주파 수가 낮을수록 더 심해지며, 잡음비가 높고, 특히 저주파 잡음 부분과 연관성이 높은 특징이 있다. ${ }^{8-10)}$ 기식음(B)은 NHR, 연 성발성지표(soft phonation index), $\mathrm{CPP}$ 등과 연관성이 있는 것으로 보고되었다. ${ }^{9-11)}$

본 연구에서 기식음은 성대결절, 성대구증에서 $\mathrm{CPP}$ 와 연 관성을 보였으며 특히 성대마비에서는 높은 연관성을 보였다. 성대마비 환자에서 주된 음성장애는 성문폐쇄부전으로 인한 기식음으로 알려져 있다. 기식음은 진동하는 성대 사이로 빠 르게 나오는 난기류에 의해 발생하며 첫 번째 배음의 강도가 두 번째 배음에 비해 과도하게 증가해서 나타난다. ${ }^{12)} \mathrm{CPP}$ 는 음성신호 중 배음양을 기반으로 전체 강도를 정규화한 강도 측정값이다. 기본주파수의 주기성과는 독립적으로 배음의 강 도를 측정하므로 기식음의 특징을 잘 반영하는 것으로 알려 져 있다. ${ }^{13-15)}$ 성문폐쇄부전에 의한 기식음은 일측성 성대마비 뿐만 아니라 성대결절이나 성대구증에서도 나타나는 일반적 인 병태생리이지만 성대마비만큼 전형적인 것은 아니므로 연 관성은 성대마비에 비해 낮게 나타나는 것으로 판단된다. 성

Table 5. Correlation between subjective and objective parameters in vocal nodule patients $(n=58)$

$\begin{array}{lllllll} & \text { VHI } & \text { G } & \text { R } & \text { B } & \text { A } & \text { S } \\ \text { CPP-a } & -0.227 & -0.605^{*} & -0.397 & -0.569^{*} & -0.219 & -0.135 \\ \text { CPP-s } & -0.327^{*} & -0.646^{*} & -0.454 & -0.500^{*} & -0.128 & -0.283^{*} \\ \text { F0 } & 0.194 & 0.284^{*} & 0.254 & 0.140 & -0.091 & -0.007 \\ \text { Jitter } & 0.136 & 0.557^{*} & 0.537^{*} & 0.460^{*} & 0.006 & 0.226 \\ \text { Shimmer } & 0.222 & 0.590^{*} & 0.546^{*} & 0.426^{*} & 0.008 & 0.312 \\ \text { NHR } & 0.097 & 0.435^{*} & 0.474 & 0.276^{*} & -0.037 & 0.387^{*} \\ \text { MPT } & -0.108 & -0.348^{*} & -0.202 & -0.321^{*} & -0.103 & -0.075 \\ \text { MFR } & 0.052 & 0.166 & 0.008 & 0.233 & -0.029 & 0.232 \\ \text { SP } & 0.017 & 0.413^{*} & 0.404^{*} & 0.351 & -0.031 & 0.446\end{array}$

${ }^{*} \mathrm{p}<0.05$. VHI: Voice Handicap Index, G: grade, R: rounghness, B: breathiness, A: asthenic, S: strained, CPP-a: cepstral peak prominence in /a/, CPP-s: cepstral peak prominence in sentence reading, F0: fundamental frequency, NHR: noise to harmonic ratio, MPT: maximal phonation time, MFR: mean flow rate, SP: subglottic pressure

Table 6. Correlation between subjective and objective parameters in unilateral vocal fold paralysis patients $(n=30)$

\begin{tabular}{lccccccc} 
& VHI & G & R & B & A & S \\
\hline CPP-a & -0.317 & $-0.754^{*}$ & -0.289 & $-0.594^{*}$ & $-0.520^{*}$ & -0.060 \\
CPP-s & -0.154 & $-0.838^{*}$ & -0.369 & $-0.706^{*}$ & $-0.611^{*}$ & -0.028 \\
F0 & 0.047 & -0.198 & 0.055 & -0.179 & -0.139 & 0.288 \\
Jitter & 0.139 & $0.660^{*}$ & 0.260 & $0.570^{*}$ & 0.266 & 0.180 \\
Shimmer & 0.176 & $0.575^{*}$ & 0.431 & $0.553^{*}$ & 0.034 & 0.300 \\
NHR & 0.217 & $0.574^{*}$ & 0.157 & $0.527^{*}$ & 0.112 & $0.526^{*}$ \\
MPT & -0.258 & $-0.752^{*}$ & -0.217 & $-0.657^{*}$ & $-0.619^{*}$ & -0.228 \\
MFR & 0.106 & $0.403^{*}$ & 0.107 & $0.538^{*}$ & 0.342 & 0.133 \\
SP & 0.230 & $0.376^{*}$ & 0.112 & $0.405^{*}$ & 0.044 & 0.222 \\
\hline
\end{tabular}

${ }^{*} \mathrm{p}<0.05$. VHI: Voice Handicap Index, G: grade, R: rounghness, B: breathiness, A: asthenic, S: strained, CPP-a: cepstral peak prominence in /a/, CPP-s: cepstral peak prominence in sentence reading, F0: fundamental frequency, NHR: noise to harmonic ratio, MPT: maximal phonation time, MFR: mean flow rate, SP: subglottic pressure 
대마비와 성대결절 그리고 정상인의 $\mathrm{CPP}$ 값을 비교한 연구에 서 정상인에 비해 성대마비, 성대결절 모두 유의하게 낮은 값 을 보였고 성대마비는 성대결절보다 더 낮은 값을 보였다. ${ }^{16)}$

거친음은 불규직한 성대진동와 기본주파수의 동요(fluctuation)에 의해 발생한다. ${ }^{17)}$ 음향학적 평가 중 주파수변동율, 진폭변동율 등은 기본주파수의 주기성(periodicity)에 영향을 받기 때문에 성대진동의 불규칙성에 의해 나타나는 거친음을 표현한다고 할 수 있다. ${ }^{18)}$ 본 연구에서는 성대구증에서 진폭 변동율, 그리고 성대결절에서 주파수변동율 및 진폭변동율이 중등도의 상관관계를 보였다. 성대점막에 발생한 성대결절이 나 성대구증에 의해 성대진동이 방해를 받아 거친음이 발생 한 것으로 판단된다. 하지만 비대칭적인 성대진동을 유발시킬 것으로 생각되는 성대용종에서는 연관성이 낮게 나타났는데 이는 성대용종이 성대결절이나 성대구증에 비해 점막물결에 대한 영향이 제한적일 가능성을 시사한다. 따라서 각 질환들 의 진동 분석을 포함한 더 많은 증례에 대한 연관성 분석이 필 요할 것으로 판단된다.

전체음질 $(\mathrm{G})$ 은 전체 환자에서뿐만 아니라 질환별로 분석하 였을 때도 가장 많은 객관적 평가 항목들과 연관성을 보이고 있으며 특히 GRBAS 척도의 다른 항목과 객관적 평가 항목 사이에 연관성에 있는 경우에 대부분의 경우에 전체음질과 동시에 연관성이 있는 것으로 나타나 전체음질이 전반적인 청지각적 음성 상태를 반영한다고 판단된다. ${ }^{19}$

음성장애지수와 객관적인 음성평가의 상관관계를 분석한 기존 연구에서는 기능적 음성장애와 성대용종 환자에서 음성 장애지수와 최대발성시간 사이에 연관성이 있으며, 성대마비 환자는 주파수변동율, 성대결절은 주파수변동율과 진폭변동 율 그리고 NHR 등과 연관성이 있는 것으로 나타났다." 반면 메타 분석에서 청지각적평가와 가장 유의미한 연관성을 보였 던 객관적 음성평가인 $\mathrm{CPP}$ 는 음성장애지수와는 중간 또는 낮은 정도의 연관성을 보였다. ${ }^{20)}$ 본 연구에서는 어떠한 객관적 인 평가 항목과도 유의한 관계를 보이지 않았다. 이는 음성장 애지수가 목소리의 상태뿐만 아니라 더 넓은 의미의 신체적, 사회적 영향까지 측정하는 검사이기 때문으로 판단된다.

공기역학적검사는 주관적인 평가 항목들과 다양한 연관성 을 보여주지는 못했지만 예상대로 전체음질, 기식음와 연관성 이 있는 것으로 나타났으며 특히 성대마비 환자에서 최대발성 시간이 높은 연관성을 보여주었다.

\section{결 론}

발성장애 환자에서 주관적인 음성평가와 객관적인 음성평 가 항목들 간의 연관성을 분석하였을 때 $\mathrm{CPP}-\mathrm{a}, \mathrm{CPP}-\mathrm{s}$, 주파
수변동율, 진폭변동율 등이 전체음질 $(\mathrm{G})$ 과 기식음 $(\mathrm{B})$ 을 반영 하는 것으로 나타났다. 특히 일측성 성대마비에서는 CPP-a,

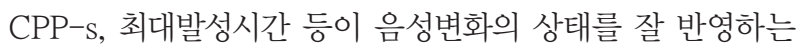
것으로 나타났다.

중심 단어: 음성, 평가, 음향학, 청지각, 연관성.

Acknowledgments

None.

Conflicts of Interest

The authors have no financial conflicts of interest.

\section{Authors' Contribution}

Conceptualization: Dong Young Kim, Joo Hyun Woo. Data curation: Boram Kim. Formal analysis: Tae Kyu Kang, Joo Hyun Woo. Investigation: Jae Hwan Oh. Writing-original draft: Jung Woo Park, Joo Hyun Woo. Approval of final manuscript: all authors.

\section{REFERENCES}

1. Dejonckere PH, Bradley P, Clemente P, Cornut G, Crevier-Buchman L, Friedrich G, et al. A basic protocol for functional assessment of voice pathology, especially for investigating the efficacy of (phonosurgical) treatments and evaluating new assessment techniques. Guideline elaborated by the Committee on Phoniatrics of the European Laryngological Society (ELS). Eur Arch Otorhinolaryngol 2001; 258(2):77-82.

2. Maryn Y, Roy N, De Bodt M, Van Cauwenberge P, Corthals P. Acoustic measurement of overall voice quality: a meta-analysis. J Acoust Soc Am 2009;126(5):2619-34.

3. V Latoszek BB, Maryn Y, Gerrits E, De Bodt M. A meta-analysis: acoustic measurement of roughness and breathiness. J Speech Lang Hear Res 2018;61(2):298-323.

4. Woisard V, Bodin S, Yardeni E, Puech M. The voice handicap index: correlation between subjective patient response and quantitative assessment of voice. J Voice 2007;21(5):623-31.

5. Hillenbrand J, Cleveland RA, Erickson RL. Acoustic correlates of breathy vocal quality. J Speech Hear Res 1994;37(4):769-78.

6. Kim JO, Lim SE, Park SY, Choi SH, Choi JN, Choi HS. Validity and reliability of korean-version of voice handicap index and voice-related quality of life. Speech Sciences 2007;14(3):111-25.

7. Schindler A, Mozzanica F, Vedrody M, Maruzzi P, Ottaviani F. Correlation between the Voice Handicap Index and voice measurements in four groups of patients with dysphonia. Otolaryngol Head Neck Surg 2009;141(6):762-9.

8. Muñoz J, Mendoza E, Fresneda MD, Carballo G, López P. Acoustic and perceptual indicators of normal and pathological voice. Folia Phoniatr Logop 2003;55(2):102-14.

9. Bhuta T, Patrick L, Garnett JD. Perceptual evaluation of voice quality and its correlation with acoustic measurements. J Voice 2004;18(3):299304.

10. de Krom G. Some spectral correlates of pathological breathy and rough voice quality for different types of vowel fragments. J Speech Hear Res 1995;38(4):794-811.

11. Heman-Ackah YD, Heuer RJ, Michael DD, Ostrowski R, Horman M, Baroody MM, et al. Cepstral peak prominence: a more reliable measure of dysphonia. Ann Otol Rhinol Laryngol 2003;112(4):324-33.

12. Klatt DH, Klatt LC. Analysis, synthesis, and perception of voice quality variations among female and male talkers. J Acoust Soc Am 1990; 87(2):820-57.

13. Awan SN, Roy N. Toward the development of an objective index of 
dysphonia severity: a four-factor acoustic model. Clin Linguist Phon 2006;20(1):35-49.

14. Awan SN, Roy N. Outcomes measurement in voice disorders: application of an acoustic index of dysphonia severity. J Speech Lang Hear Res 2009;52(2):482-99.

15. Kim TH, Choi JI, Lee SH, Jin SM. Comparison of vowel and text-based cepstral analysis in dysphonia evaluation. J Korean Soc Laryngol Phoniatr Logop 2015; 26(2):117-21.

16. Park MC, Mun MK, Lee SH, Jin SM. Clinical usefulness of cepstral analysis in dysphonia evaluation. Korean J Otorhinolaryngol-Head Neck Surg 2013:56;574-8.

17. Dejonckere PH, Lebacq J. Acoustic, perceptual, aerodynamic and anatomical correlations in voice pathology. ORL J Otorhinolaryngol Relat Spec 1996;58(6):326-32.

18. Dejonckere PH, Remacle M, Fresnel-Elbaz E, Woisard V, Crevier-Buchman L, Millet B. Differentiated perceptual evaluation of pathological voice quality: reliability and correlations with acoustic measurements. Rev Laryngol Otol Rhinol (Bord) 1996;117(3):219-24.

19. Eadie T, Sroka A, Wright DR, Merati A. Does knowledge of medical diagnosis bias auditory-perceptual judgments of dysphonia? J Voice 2011;25(4):420-9.

20. Awan SN, Roy N, Cohen SM. Exploring the relationship between spectral and cepstral measures of voice and the Voice Handicap Index (VHI). J Voice 2014;28(4):430-9. 